\title{
Enhancedcongestion Control In Reset Frequency Controlled Decp_Tfrc
}

\author{
Dr. B. Chellaprabha ${ }^{1}$, M. Abinaya ${ }^{2}$, V. Chithra ${ }^{3}$, P. Gnanaprabha ${ }^{4}$ \\ ${ }^{1}$ Head, Department of computer science and engineering, SNS College of Engineering, India \\ ${ }^{2,3,4}$ Student, Department of computer science and engineering, SNS College of Engineering, India \\ India
}

\begin{abstract}
Congestion control (CC) is one of the major issues in wireless sensor network. CC will be handled in Data link layer, Network layer and Transport layer. Among all existing transport protocols, it is proved that DCCP is performing better than all protocols compared. In DCCP, there are two congestion control identifier (CCID)-TCP_LIKE (CCID2), TCP_TFRC (CCID 3) In the proposed system, DCCP_TFRC is considered. Reset is one of the main functions in DCCP which resets all the parameters to its default values when it is invoked. Whenever the connection is to be closed for some reason the reset function is invoked. If congestion occurs in DCCP-TFRC, the reset function is called and parameters involved in this protocol are set to their default values. In the existing RC_DCCP_TFRC, when the reset function is called for congestion instead of setting the parameters to their default values, it sets the selected parameters with the recent values observed. In this proposed work, some more parameters are selected along with existing parameters in RC DCCP-TFRC to analyze the performance. Three metrics namely

Throughput, Average Delay, Energy Consumption was considered in this work. Results show that there is a slight improvement in all the metrics considered.
\end{abstract}

Key words: Congestion Control, Transport Protocols, Sensor Networks, DCCP, DCCP_TFRC

\subsection{The Wireless Sensor Network (WSN)}

\section{Introduction}

A wireless sensor network refers to a group of spatially dispersed and dedicated sensors for monitoring and recording the physical conditions of the environment and organizing the collected data at a central location. Wireless sensor network monitor physical or environmental conditions, such as temperature, sound, vibration, pressure, motion or pollutants at different locations and to cooperatively pass their data through the network to a main location. Each device in a wireless sensor network is termed as node and each node is equips with wireless communication device, typically a transceiver, a microcontroller and an energy source. Wireless sensor network transmission is multi hop in nature and comprised of energy constrained nodes.

The information sensed by sensor devices are sent in form of data packets and for the reliable communication the transport layer protocol is embedded between application layer and network layer. The topology of WSNs can vary from a simple star network to an advanced multi-hop wireless mesh network. The propagation technique between the hops of the network can be routing or flooding.

\section{2need Of This Work}

The typical wireless sensor network is highly unstable as it is error-prone due to interference of radio signal, radio channel contention and survival rate of nodes. Comparatively new protocol DCCP having interesting properties, which makes it possible to use it in an error- prone sensor network. DCCP is a very good candidate for a replacement protocol for other existing protocol in the transport layer. The satisfactory performance of the DCCP has been established by the authors in their previous work. However, in a detailed analysis that followed, it was felt that there are certain aspects of DCCP need to be improved further to make it more suitable for WSN. As it was found that the reset function and timeout parameters have considerable influence on reporting interval and overall performance of sensor network, it is proposed to exploit those parameters to implement DCCP in WSNs more efficiently.

\section{Datagram Congestion Control Protocol (Dccp)}

With User Datagram Protocol (UDP) as the base, DCCP is developed for effective and efficient handling of congestion. The main objective of DCCP is to extend support for implementing different congestion control schemas. Among the applications the multimedia streams is selected to provide efficient congestion control. DCCP over WSN resulting in more reliable transmission of data grams or packets. In DCCP the bandwidth utilization is improved by increasing the data packet size to the header size ratio. 
Congestion control is implemented in DCCP_TFRC by varying the transmission rate using additive increase and subtractive decrease and this technique of congestion control is known as CCID3. DCCP_TFRC is a received based congestion control mechanism that provides a TCP-friendly sending rate while reducing the abrupt-rate-change characteristic of TCP or TCP-Like congestion control.

\section{Reset Frequency Controlled Parameter Re-Estimation Method}

Reset function is frequently called in several states of the client server communication process during the typical DCCP communication scenario. A DCCP-Reset can be initiated by both server and client and a DCCP agent resets the connection if any feature negotiation fails during the handshake. After receiving the reset request in any state the server moves to LISTEN state and client moves to CLOSED state irrespective of the current state. In DCCP protocol reset function is called often to avoid congestion. During each reset, all the parameters are set to their initial values to reduce the overhead and congestion.

\section{Modifications in DCCP Reset Function}

The parameter considered in the previous works are rate at which a sender can transmit data packets per second-send_rate (s_x), current send rate-s_x_inst, during that a sender has to wait before retransmissioninitial_rtx_to and the maximum retransmission time - max_rtx-to. In addition to that, the parameter congestion window- CWindow is added for the modification. These parameters are updated for every occurrence of a reset call, which means that if there is less congestion in the network the sender will transmit more packets by increasing the send rate and the receive rate. A parameter Average Reset Inter is computed at each stage by taking the average of the accumulated average of preceding reset intervals and the current reset interval. In this context, congestion window is calculated at each interval.

An increase in $t_{r r}$ indicates less congestion in the network due to which the average data packet reaching the destination increases and also the time taken for each packet to reach the receiver get decreases. So that the acknowledgement from a receiver will reach the sender relatively earlier compared to high congestion state.

In contrast, when $t_{r r}$ decreases then there will be rush of packets which leads to higher congestion and the algorithm behaves similar to normal DCCP_TFRC and all the parameters will be set to their initial values. As the proposed algorithm updates the parameters depending on the frequency of the reset function call this is named as Enhanced Reset-frequency Controlled DCCP_TFRC

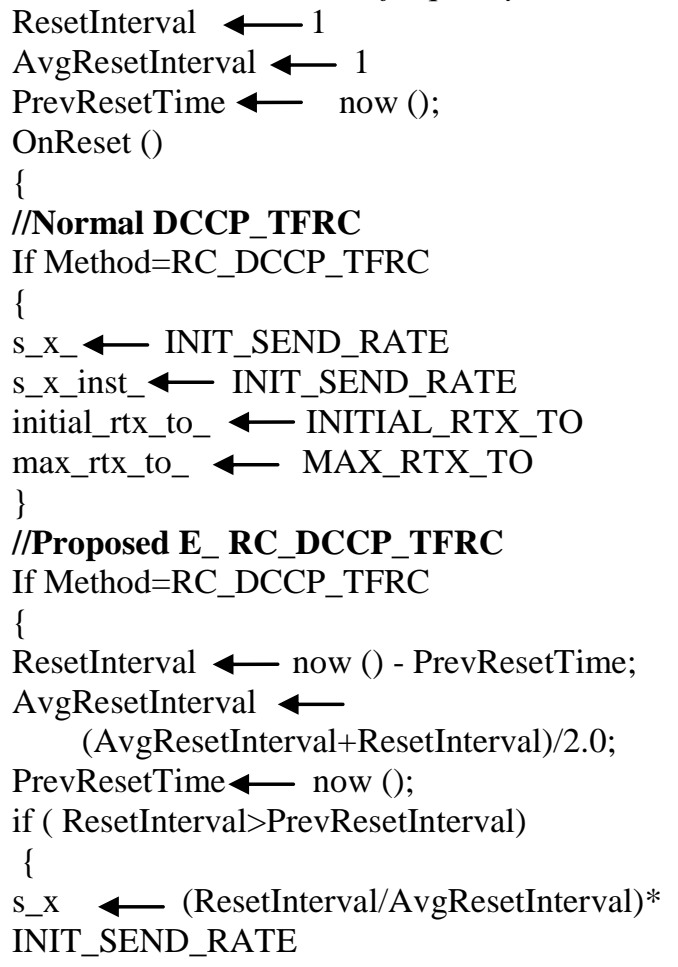




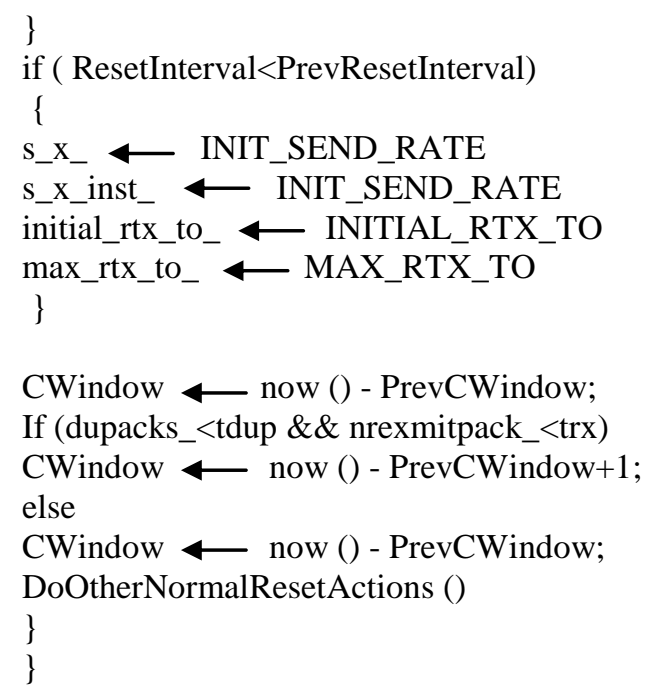

The above modification will reset the initial values with respect to the state of the congestion control algorithm. If reset is called during the high congestion then the ResetInterval will be greater than the AvgResetInterval. Similarly, the reset is called at a low congestion state, and then the ResetInterval will be lower than the AvgResetInterval. In this work, the duplicate acknowledgement and the number of retrainsmission packets are calculated. If the duplicate acknowledgement is lesser than the threshold duplicate acknowledgement and the number of retransmission packet is lower than the threshold retransmission, then the congestion window size is reduced. whenever the congestion window is reduced, the receive rate will be increased that is the vice versa. So that the initial parameters will be reset according to the congestion state. If the values are higher than the threshold values, the congestion window is increased and the receive rate will be decreased. Now the system works like normal DCCP_TFRC.

\section{Result and Analysis}

The RC_DCCP_TFRC is simulated with different data reporting intervals repeatedly and their performance is analyzed using the metrics throughput, Energy consumption and Average Delay.

It is measured, plotted and discussed in the following section.

\subsection{Metrics considered for Evaluation}

\subsubsection{Throughput}

The rate of data packet arrival with respect to time at the destination or sink is termed as the throughput of the network. It is noticed that the performance of the proposed E_RC_DCCP_TFRC with shows better performance than the RC_DCCP_TFRC.

\section{Numberofpackets Vs Time (Sec)}

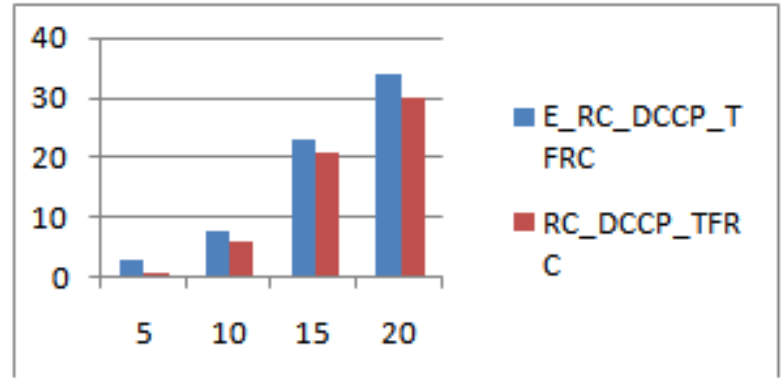

\subsubsection{Energy Consumption}

The average energy consumed by all the nodes of the network is considered as a metric to assess the performance of the protocol. It is found that the total energy consumed during data transmission for proposed E_RC_DCCP_TFRC is less when compared to the RC_DCCP_TFRC protocol.

ENERGY (joules) Vs TIME (sec) 


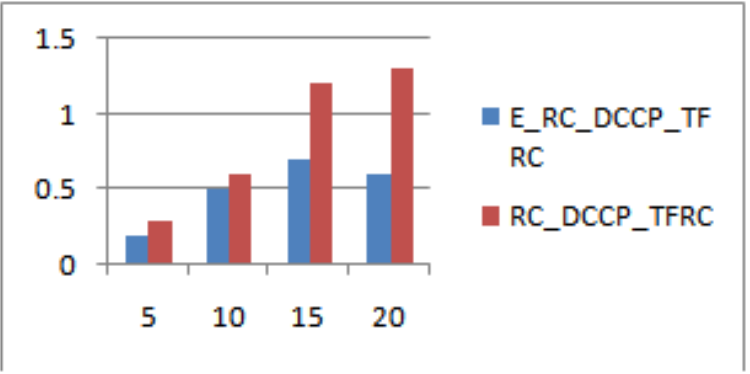

\subsubsection{Average Delay}

Average delay is the cumulative delay that might come about as a result of buffering during discovery of routes over sensor network. It is observed that the delay is lesser in the proposed E_RC_DCCP_TFRC and is higher in the RC_DCCP_TFRC.

\section{DELAY VS TIME (sec)}

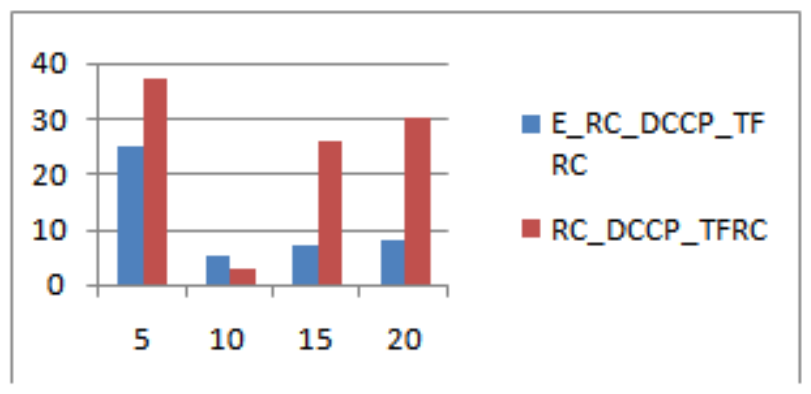

\section{Conclusion}

To improve the performance of WSNs based on DCCP protocols during congestion, the shortfalls were analyzed and some changes were proposed during reset action that takes place during congestion. The proposed E_RC_DCCP_TFRC shows the better performance when compared to the RC_DCCP_TFRC with respect to the metrics throughput, energy consumption and average delay. In the future work, other additional parameters can be considered for the evaluation of the E_RC_DCCP_TFRC.

\section{References}

[1]. B.Chellaprabha, Dr.S.ChenthurPandian, Dr. C. Vivekanandan, "Reset frequency controlled parameter Re-estimation method for the improvement congestion control in DCCP_TFRC".

[2]. B.Chellaprabha, Dr.S.ChenthurPandian, Dr. C. Vivekanandan, "Performance of TCP, UDP and SCTP on Sensor Network with Different Data Reporting Intervals", IOSR Journal of Engineering, Apr. 2012, Vol. 2(4) pp: 621-628

[3]. B.Chellaprabha, Dr.S.ChenthurPandian, Dr. C. Vivekanandan, "Performance of Datagram Congestion Control Protocol DCCPTCPLike and DCCP-TFRC on Sensor Network", IRACST - International Journal of Computer Networks and Wireless Communications (IJCNWC), ISSN: 2250-3501, Vol.2, No.2, April 2012.

[4]. B.Chellaprabha, Dr.S.ChenthurPandian, Dr. C. Vivekanandan, "An Extensive Evaluation of Transport Protocols Under Highly Congested Sensor Network", European Journal of Scientific Research ISSN 1450-216X, Vol.78, No.1,2012, pp.93-106

[5]. [5] Jang-Ping Sheu, Li-Jen Chang and Wei-Kai Hu, "Hybrid Congestion Control Protocol in Wireless Sensor Networks", Journal Of Information Science And Engineering 25, pp. 1103-1119, 2009.

[6]. Y. G. Iyer, S. Gandham, S. Venkatesan, "STCP: a generic transport layer protocol for wireless Sensor networks", 14th International Conference on In Computer Communications and Networks, pp. 449-454, 2005.

[7]. Alam and Hong, "Congestion-Aware and Rate-Controlled Reliable Transport in WSNS", The Institute of Electronics, Information and Communication Engineers, 2009.

[8]. Yao-Nan Lien, "Hop-by-Hop TCP for Sensor Networks", International Journal of Computer Networks \& Communications IJCNC, Vol.1, No.1, April 2009.

[9]. Jang-Ping Sheu1, Li-Jen Chang And Wei-Kai Hu, "Hybrid Congestion Control Protocol in Wireless Sensor Networks", Journal of information science and engineering 25, 1103-1119,2009.

[10]. Paulo Rogério Pereira, et.al. "End-To-End Reliability in Wireless Sensor Networks: Survey and Research Challenges", EuroFGI Workshop on IP QoS and Traffic Control P. Pereira (Ed.), Lisbon, Portugal, December 6-7, 2007.

[11]. Md. AbdurRahman, Abdulmotaleb El Saddik and Wail Gueaieb, "Wireless Sensor Network Transport Layer: State of the Art", Sensors, Springer-Verlag Berlin Heidelberg, pp. 221-245, 2008

[12]. B. Hull, K. Jamieson, and H. Balakrishnan, "Mitigating Congestion in Wireless Sensor Networks", SenSys '04: Proc. 2nd International Conference on Embedded Networked Sensor Systems, pp.134-147, ACM, 2004.

[13]. Chonggang Wang1, KazemSohraby, Victor Lawrence, Bo Li, Yueming Hu, "Priority-Based Congestion Control in Wireless Sensor Networks", Proceedings of the IEEE International Conference on Sensor Networks, Ubiquitous, and Trustworthy Computing SUTC'06, 2006 
[14]. D StanimirStatev, SeferinMirtchev, "Experimental Study of DCCP Transport Protocol in Varied Network States", International Scientific Conference Computer Science'2008.

[15]. Kohler E., S.FloydKohler E., S. Floyd, and M. Handley, "Designing DCCP: Congestion Control without reliability," in Proc. ACM SIGCOMM, Pisa, Italy, 2006.

[16]. Floyd S. and E. Kohler, "Profile for Datagram Congestion Control Protocol (DCCP) Congestion Control ID 2: TCP- like Congestion Control," RFC 4341 (Pro- posed Standard), Mar. 2006.

[17]. Floyd S., E. Kohler, and J. Padhye, "Profile for Datagram Congestion Control Protocol (DCCP) Congestion Control ID 3: TCPFriendly Rate Control (TFRC)," RFC 4342 (Proposed Standard), Mar. 2006.

\section{Authors Profile}

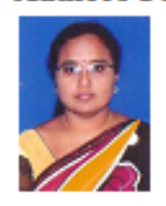

Dr. B. Chellaprabha completed her B.Sc degree from Bharathidasan University in 1993, Master of Computer Applications from Bharathidasan University in 1996 and M.E in Computer Science and Engineering Anna University Chennai in 2007. She has completed doctoral degree in Anna University of Technology, Coimbatore. She has more than 15 years of teaching experience. She is working as a Professor and Head of the Department of Computer Science and Engineering, SNS College of Engineering, Coimbatore, Tamil Nadu. Her areas of interest include Wireless sensor networks, Routing protocols, Congestion control and detection and Network Security.

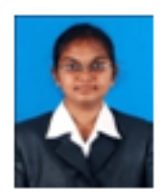

M.Abinaya is currently pursuing her bachelor of Engineering in the stream of Computer science and engineering in SNS college of engineering, Coimbatore, India.

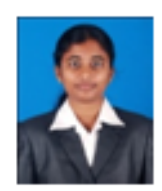

V.Chithra is currently pursuing her bachelor of Engineering in the stream of Computer science and engineering in SNS college of engineering, Coimbatore, India.

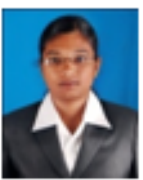

P. Gnanaprabhais currently pursuing her bachelor of Engineening in the stream of Computer science and engineering in SNS college of engineering, Coimbatore, India. 